\title{
EFFECT OF TOBACCO EXTRACT ON STREPTOCOCCUS MUTANS: POSSIBLE ROLE IN MODULATING CARCINOGENESIS
}

\begin{abstract}
JYOTSNA ARUN ${ }^{1}$, SRIKANT N ${ }^{1 *}$, ETHEL SUMAN ${ }^{2}$, ASHOK SHENOY $^{3}$, SRIKALA BALIGA ${ }^{2}$
${ }^{1}$ Department of Oral Pathology and Microbiology, Manipal College of Dental Sciences, Manipal Academy of Higher Education, Mangalore, Karnataka, India. ${ }^{2}$ Department of Microbiology, Kasturba Medical College, Manipal Academy of Higher Education, Mangalore, Karnataka, India. ${ }^{3}$ Department of Pharmacology, Kasturba Medical College, Manipal Academy of Higher Education, Mangalore, Karnataka, India. Email: srikant.n@manipal.edu
\end{abstract}

Received: 22 Febuary 2018, Revised and Accepted: 18 April 2018

\section{ABSTRACT}

Objective: Tobacco use in the smoking or smokeless form is the most common form of substance abuse recorded in the world. Not only does tobacco influence carcinogenesis but also modifies the oral microflora. The aim of our study was to assess the growth pattern of Streptococcus mutans under the influence of cigarette extract.

Methods: Pure stock culture of $S$. mutans was grown in brain heart infusion broth mixed with three concentrations of aqueous cigarette extract. Quantification of the S. mutans colonies was performed in Mitis Salivarius Agar subculture. Biofilm assessment was also performed to find the adherent property of microorganisms.

Statistical Analysis used: One-way ANOVA was used to compare the effect of cigarette extract on growth and biofilm formation of S. mutans.

Results: There was increase in the colony counts with increasing concentration of cigarette extract $(p<0.001)$. There was an observable trend noted in the biofilm assay.

Conclusion: The colony growth is positively influenced by the cigarette additives (sugars and sweeteners), carbon dioxide environment, and biofilm modification. The altered flora with higher S. mutans may be linked with the higher salivary acetaldehyde production which promotes carcinogenesis. The flora may be protective by production of antitumorigenic or antimutagenic compounds. The balance between the carcinogenic and anticarcinogenic signals produced by tobacco and microflora influences the outcome of the disease.

Keywords: Tobacco extract, Streptococcus mutans, Carcinogenesis, Mutagenesis.

(C) 2018 The Authors. Published by Innovare Academic Sciences Pvt Ltd. This is an open access article under the CC BY license (http://creativecommons. org/licenses/by/4. 0/) DOI: http://dx.doi.org/10.22159/ajpcr.2018.v11i7.25425

\section{INTRODUCTION}

Substance abuse is one of the biggest curses that modern society has come across. It has widely afflicted the globe. Of the various substances abused, the most widely distributed and the most commonly used is "tobacco." Over the past four decades, tobacco use has caused an estimated 12 million deaths in the world including 4.1 million from cancer, 5.5 million from cardiovascular system diseases, 2.1 million deaths from respiratory diseases, and 94,000 infants' deaths related to mothers smoking during pregnancy. The $50^{\text {th }}$ round of survey by National Sample Survey Organisation, NSSO, in India, showed that $51.3 \%$ males and $10.3 \%$ of females are regular tobacco users, $35.3 \%$ males and $2.6 \%$ females were regular smokers, and $24 \%$ males and $8.6 \%$ females were regular users of smokeless tobacco, and about 250 million users were aged $10+$ years in the country [1]. Another survey by National Family Health Survey, NFHS, in its $2^{\text {nd }}$ round revealed that tobacco use among men was $46.5 \%$ and among women, $13.8 \%$ [2].

Today, the most common method of using tobacco is in manufactured cigarettes.

Apart from being a known carcinogen, tobacco is known to cause change in the microflora by providing a sugar substrate conducive to growth of cariogenic bacteria. It also increases the carbon dioxide concentration that favors the growth of Streptococcus mutans which, in turn, leads to various oral health issues such as staining, halitosis, periodontitis, and dental caries.
The aim of our study was to assess the growth pattern of S. mutans under the influence of cigarette extract.

\section{METHODS}

Preparation of the extract

Water extract of cigarettes was made using Soxhlet extraction technique (Fig. 1). The cigarette contents were first wetted in water and then introduced into Soxhlet apparatus where it passes through the condenser, porous container, and distilling pot in order. The extract so obtained was filter sterilized using a membrane filter, and cigarette extract was collected and refrigerated. This water extract was mixed with brain heart infusion (BHI) broth to obtain three concentrations of $1: 12.5,1: 25$, and 1:50 (indicating the broth to water extract ratio).

\section{Preparation of culture}

Pure strains of $S$. mutans maintained at the Department of Microbiology, KMC, Mangalore, were obtained and cultured in BHI broth. $2 \mathrm{ml}$ of this pure culture was added to broth with concentrations of the cigarette extract in triplets (three for each concentration) and incubated for 48 $\mathrm{h}$ at $37^{\circ} \mathrm{C}$. These samples were further subcultured on Mitis Salivarius Agar and incubated for $72 \mathrm{~h}$ at $37^{\circ} \mathrm{C}$ following which the colonies of S. mutans were quantified.

\section{Preparation of biofilm}

About $0.5 \mathrm{ml}$ of $S$. mutans culture was transferred to 96-well microtiter plate to which $0.5 \mathrm{ml}$ of cigarette extract was added and then incubated 
for $24 \mathrm{~h}$ at $37^{\circ} \mathrm{C}$ for biofilm formation. This was assessed by staining with $1 \%$ crystal violet, and the absorbance was evaluated using ultraviolet spectrophotometer at $540 \mathrm{~nm}$ wavelength of light. Following this, the biofilm adherence score was read and tabulated (Figs. 1 and 2a-c). The biofilm adherence were assessed with different concentration of cigarette extract.

\section{RESULTS}

On comparison of the mean colony counts of the $S$. mutans with different dilutions of cigarette extract using one-way ANOVA, we found that growth of $S$. mutans had a positive correlation with the concentration of cigarette extract $(\mathrm{p}<0.001)$. Higher concentration

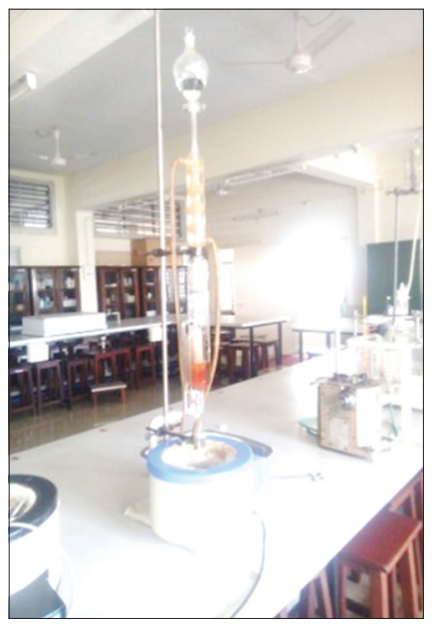

Fig. 1: Soxhlet apparatus used to prepare an aqueous extract of tobacco from cigarette

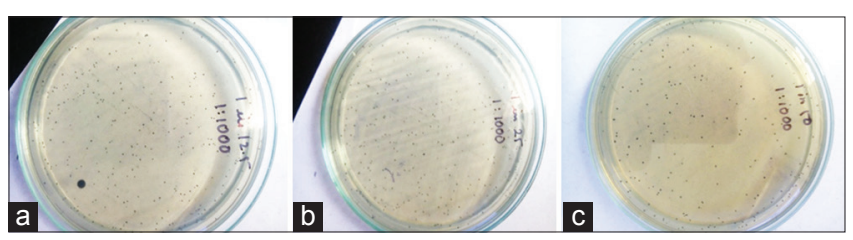

Fig. 2: Growth of Streptococcus mutans in different concentrations of tobacco extract. (a) 1:12.5, (b) 1:25, (c) 1:50 concentration.

Note the progressive reduction in the colony growth as the concentration of tobacco extract decreases (from a to c)
(1:12.5) showed higher colony counts of $10.5 \times 10^{-6}$. The dilution of $1: 25$ showed a reduction of colony to nearly half than at $1: 12.5\left(4.585 \times 10^{-6}\right)$ and at further dilution of $1: 50$ it dropped down to $2.1 \times 10^{-6}$ colonies (Fig. 3 ).

Assessment of the spectrophotometric readings of the biofilm showed that the results varied in each concentration of cigarette extract. In 1:10 dilution, higher adhesion was observed, in 1:100 dilution, reduced adhesion was observed while 1:1000 dilution showed similar adhesion property as that of 1:100 dilution (Fig. 4).

\section{DISCUSSION}

Various researches have established that cigarette smoking greatly affects the oral microflora. Oral microflora alters not only the oral hygiene status but also influences the development of diseases such as dental caries and oral cancer.

In our study, we assessed the growth of the S. mutans under the influence of tobacco extract and found a significant increase in the colonies with higher concentration of the tobacco extract due to a variety of reasons. Colony count of the cariogenic microorganisms increases due to contents of the cigarette or modifications in oral environment induced by cigarette smoking.

The first reason for colony increase could be attributed to the presence of sugar content in cigarette extract which provides favorable conditions for increase in growth of cariogenic microorganisms (S. mutans). Tomar and Winn (1999) in their study showed that three popular brands of smokeless tobacco had $26 \%$ by weight of fermentable sugar [3]. Vellapally et al. (2007) reviewed the influence of tobacco on caries and highlighted the presence of various sugars and sweeteners during manufacture of cigarettes which could be the reason for increased growth of cariogenic microorganisms under the influence of cigarette extract [4].

The second reason for higher colony counts associated with tobacco use could be attributed to increased carbon dioxide concentration. Zonuz et al. (2008) suggested that cigarette smoking increases carbon dioxide concentration which favors the growth of $S$. mutans. It was observed that there was 18-40\% increase in growth of cariogenic microbes owing to cigarette smoking [5]. Another study by Ebrahimi et al. (2014) found that cigarette smoke induces microaerophilic environment which increases the growth of $S$. mutans by $81.95 \%$ [6].

Huang et al. (2012) proposed that the reason for colony increase could be attributed to increased biofilm formation by $S$. mutans. They found out that smoking increase the formation of $S$. mutans biofilm on tooth

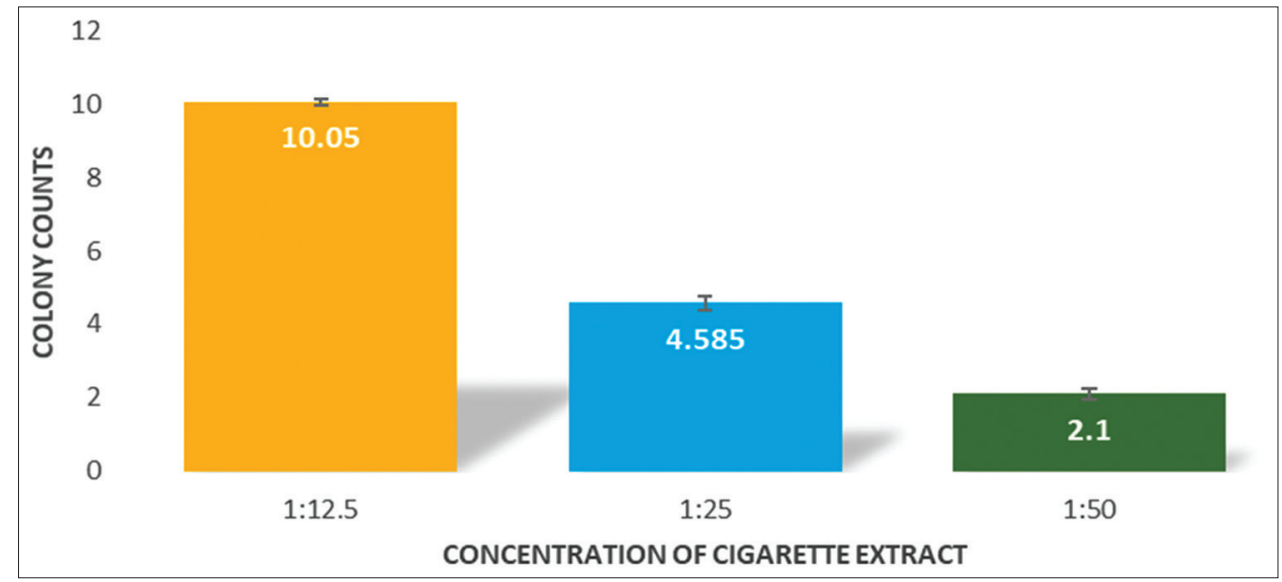

Fig. 3: Mean Streptococcus mutans counts are seen to decrease significantly with decrease in the concentration of cigarette extract (1:12.5 is the most concentrated and 1:50 is the most diluted) 


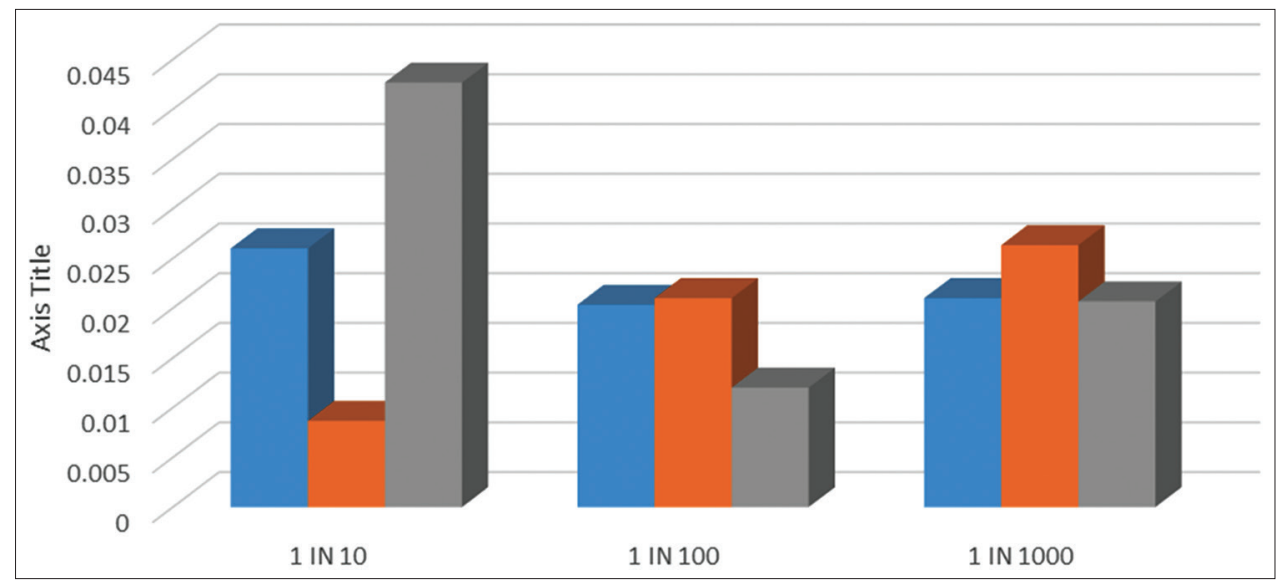

Fig. 4: The biofilm formation seen at three dilutions of 1:10, 1:100, and 1:1000 at three concen-trations of cigarette extract

surface, which increased the development of caries due to increased lactic acid production owing to enhanced metabolism of S. mutans [7]. Although in our study, we did not find a significant association of a definitive trend in the biofilm and tobacco usage, biofilm formation is an important factor that needs further assessment.

Tobacco in smoking and smokeless forms is the main causes of cancer of the oral cavity and upper gastrointestinal tracts and accounts for nearly $80 \%$ of the cases [8]. Epidemiological reports and studies have shown that other factors related to dentition such as tooth loss, poor dental status, and oral hygiene habits are associated with higher risks, for the development of oral cavity cancer $[9,10]$.

Experiments on cell cultures and animal models have brought forth evidence that acetaldehyde production by the oral microorganisms in the oral cavity influences carcinogenesis. Under the influence of tobacco products, a "switch" of the oral flora toward Gram-positive (acetaldehyde producing) organisms from Gram-negative bacteria has been described in smokers which can further contribute to the carcinogenesis [11]. Homann et al. (2000) have found that smoking increased the salivary acetaldehyde production by $50-60 \%$ [12]. This could also be attributed to the qualitative as well as quantitative changes of the oral microflora induced by smoking.

Tobacco can directly or indirectly cause this alteration in oral microflora. Tobacco contains numerous phytochemicals, one such chemical nitrogen-containing alkaloids have properties of blocking enzyme action [13]. Processed tobacco also contains sweeteners and additives like nutmeg butter (derived from Myristica fragrans, nutmeg tree), which is known to have inhibitory activity against several kinds of anaerobic and aerobic microorganisms [14].

Oral microflora-induced suppression of tumor is also reported in the literature. S. mutans is a normal commensal of the oral cavity and is suggested as the main cariogenic bacteria. A study by Tezal (2013) has shown inverse correlation of dental caries and head and neck cancer. This could be attributed to the normal commensals of the oral cavity. The mechanisms which could protect the host from the cancer include antitumorigenic or antimutagenic compounds produced by the bacteria, regulation of cytokine production, production of antimicrobial substances, clearance, inhibition of growth and downregulation of fimbrial expression of Gram-negative bacteria, production of surfactants, and stabilization of low $\mathrm{pH}[15,16]$.

\section{CONCLUSION}

In our study, we found that there is definite modification of oral microflora under the influence of tobacco extracts. The increase in the S. mutans is directly proportional to the amount of cigarette extract owing to the artificial sweeteners added during the processing. Research has shown that the altered microflora influences mechanism of tobacco-induced mutagenesis $[12,13]$. The balance between the tobacco-induced mutations, microflora-induced acetaldehydes, and the antitumoral characteristics of microflora decides the outcome of the disease in the individual.

\section{AUTHOR'S CONTRIBUTION}

The research protocol was conceived and developed by JA and SN in consultation with ES, AS, and SB. The tobacco extraction was done by JA and AS. The microbial culture and analysis were performed and supervised by JA, ES, and SB. The statistical analysis was performed by SN. The manuscript writing, editing, and revisions were performed by $\mathrm{JA}, \mathrm{SN}$, and ES.

\section{CONFLICTS OF INTEREST}

The authors have no conflict of interest to be declared for the present research work. This work is self-funded with no external source of funding.

\section{REFERENCES}

1. National Sample Survey Organization (NSSO). Fifty Second Round of National Sample Survey on Household Expenditure (1997). New Delhi: Ministry of Statistics and Programme Implementation, Government of India; 1997.

2. International Institute for Population Sciences (IIPS), Macro International. National Family Health Survey (NFHS-2), 1998-99. Vol. 01. India, Mumbai: IIPS; 2000.

3. Tomar SL, Winn DM. Chewing tobacco use and dental caries among U.S. Men. J Am Dental Assoc 1999;130:1601-10.

4. Vellapally S, Fiala Z, Smejkalova J, Jacob V, Shriharsha P. Influence of tobacco use in dental caries development. Cent Eur J Public Health 2007; 15:116-21.

5. Zonuz AT, Rahmati A, Mortazavi H, Khashabi E, Zadeh Farahani RM. Effect of cigarette smoke exposure on the growth of Streptococcus mutans and Streptococcus sanguis: An in vitro study. Nicotine Tobacco Res 2008; 10:63-7.

6. Ebrahimi H, Bazargani A, Shahidi SP, Karimi F, Ansarifard E. The comparison of the in vitro effects of cigar and cigarette smoke on growth of Streptococcus mutans and Streptococcus sanguis. Elixir Human Physiol 2014;54:12625-30.

7. Huang $\mathrm{R}, \mathrm{Li} \mathrm{M}$, Gregory RL. Effect of nicotine on growth and metabolism of Streptococcus mutans. Eur J Oral Sci 2012;120:319-25.

8. La Vecchia, C. Tavani, A. Levi FS, Corrao G, Negri E. Epidemiology and prevention of oral cancer. Eur J Cancer Oral Oncol 1997;33:302-12.

9. Marshall JR, Graham S, Haughey BP, Shedd D, O'Shea R, Brasure J, et al. Smoking, alcohol, dentition and diet in the epidemiology of oral cancer. Eur J Cancer B Oral Oncol 1992;28B:9-15.

10. Zheng T, Boyle P, Hu H, Duan J, Jiang P, Ma D, et al. Dentition, oral hygiene, and risk of oral cancer: A case-control study in Beijing, 
People's Republic of China. Cancer Causes Control 1990;1:235-41.

11. Bardell D. Viability of six species of normal oropharyngeal bacteria after exposure to cigarette smoke in vitro. Microbios 1981;32:7-13.

12. Homann N, Tillonen J, Meurman JH, Rintamäki H, Lindqvist C, Rautio $\mathrm{M}$, et al. Increased salivary acetaldehyde levels in heavy drinkers and smokers: A microbiological approach to oral cavity cancer. Carcinogenesis 2000;21:663-8.

13. Beulah KC, Devi AT, Waseem K, Hedgekatte R, Prasad NM, Dhanjaya BL, et al. Phyto-Antiquorumones: Aan herbal approach for blocking bacterial trafficking and pathogenesis. Int J Pharm Pharm Sci 2015;7:29-34

14. Nagja T, Vimal K, Sanjeev A. Myristica fragrans: Comprehensive review. Int J Pharm Pharm Sci 2016;8:27-30.

15. Tezal M. Dental caries and head and neck cancers. JAMA Otolaryngol Head Neck Surg 2013;139:1054-60.

16. Rafter J. Lactic acid bacteria and cancer: mechanistic perspective. Br J Nutr 2002;88:S89-94. 